\title{
Capacidade para o trabalho e qualidade de vida de trabalhadores atendidos na atenção primária
}

\author{
Work ability and quality of life of workers attended in primary care
}

\section{Capacidad para el trabajo y calidad de vida de trabajadores atendidos en la atención}

Recebido: $11 / 11 / 2017$

Aprovado: 07/07/2018

Publicado: 27/09/2018
Fabiana Caetano Martins Silva e Dutra1

Alline Alves de Sousa ${ }^{2}$

Patrícia Maria Vieira ${ }^{3}$

Edna Aparecida Carvalho Pacheco 4

Rosimár Alves Querino 5

Ailton de Souza Aragão 6

O objetivo desta pesquisa foi avaliar a capacidade para o trabalho e a qualidade de vida de trabalhadores atendidos pela rede pública municipal de saúde de Uberaba-MG, e identificar possíveis associações entre capacidade para o trabalho, qualidade de vida e características sociodemográficas, econômicas e ocupacionais. Estudo transversal, envolvendo 111 trabalhadores avaliados com um questionário sobre características sociodemográficas, econômicas e ocupacionais; SF-36 para avaliação de qualidade de vida e do Índice de Capacidade para o Trabalho, no período de março a outubro de 2013. Para análise dos dados empregou-se estatística descritiva, teste MannWhitney e correlação de Spearman. A amostra foi composta em sua maioria por mulheres, com menos de 44 anos e ativas no trabalho. A capacidade para o trabalho apresentou associação significativa com idade, escolaridade, tempo de afastamento do trabalho, e com todos os domínios da qualidade de vida. Sugere-se ações de preservação da capacidade do indivíduo no trabalho e melhorias na qualidade de vida.

Descritores: Qualidade de vida;Avaliação da capacidade de trabalho; Saúde do trabalhador.

The objective of this research was to evaluate the ability for work and the quality of life of workers attended by the public health network in Uberaba-MG, Brazil and to identify possible associations between work ability, quality of life, and sociodemographic, economic and occupational characteristics. Cross-sectional study, with 111 workers who were evaluated with a sociodemographic, economic and occupational questionnaire; SF-36 for the evaluation of quality of life and the Work ability Index, from March to October 2013. Descriptive statistics, the Mann-Whitney test and Spearman correlation were used to analyze data. The sample was made mostly of women, under 44 years of age and active in the work market. Work ability had significant associations to age, educational level, number of years away from work, and all domains of quality of life. This study suggests actions to preserve the work ability of the individual and improve their quality of life.

Descriptors: Quality of life; Work capacity evaluation; Occupational health.

El objetivo de esta investigación fue evaluar la capacidad para el trabajo y calidad de vida de trabajadores atendidos por la red pública municipal de salud de Uberaba-MG, Brasil e identificar posibles asociaciones entre capacidad para el trabajo, calidad de vida y características sociodemográficas, económicas y ocupacionales. Estudio transversal, envolviendo 111 trabajadores evaluados con un cuestionario sobre características sociodemográficas, económicas y ocupacionales; SF-36 para evaluación de calidad de vida y el Índice de Capacidad para el Trabajo, en el periodo de marzo a octubre de 2013. Para análisis de los datos se empleó estadística descriptiva, test Mann-Whitney y correlación de Speraman. La muestra fue compuesta en su mayoría por mujeres, con menos de 44 años y activas en el trabajo. La capacidad para el trabajo presentó asociación significativa con edad, escolaridad, tiempo de licencia del trabajo y con todos los dominios de la calidad de vida. Se sugieren acciones de preservación de la capacidad del individuo en el trabajo y mejorías en la calidad de vida.

Descriptores: Calidad de vida, Evaluación de capacidad de trabajo; Salud laboral.

1. Terapeuta Ocupacional. Mestre e Doutora em Ciências da Reabilitação. Professora do Programa de Pós-Graduação em Atenção à Saúde e do Departamento de Terapia Ocupacional da Universidade Federal do Triângulo Mineiro (UFTM), Uberaba, MG, Brasil. ORCID: 0000-0003-3295-1583 E-mail: fabiana.dutra@uftm.edu.br

2. Psicóloga do Centro de Referência em Saúde do Trabalhador - Regional Uberaba, MG, Brasil. ORCID: 0000-0002-4643-8089 E-mail: allineasousa@hotmail.com

3. Nutricionista. Especialista em Metodologia do Ensino Superior. Mestre em Ciências da Saúde. Doutora em Alimentos e Nutrição. ProfessoraAdjunta do Departamento de Nutrição da UFTM, Uberaba, MG, Brasil. ORCID: 0000-0002-9963-5465 E-mail: patricia.vieira@uftm.edu.br

4. Assistente Social. Mestre e Doutora em Serviço Social. Professora Adjunta do Departamento de Serviço Social da UFTM, Uberaba, MG, Brasil. ORCID: 0000-0002-8130-1985 E-mail: ednacarvalhopacheco@gmail.com

5. Cientista Social. Mestre e Doutora em Sociologia. Pós Doutora em Ciências da Saúde. Professora Associada do Departamento de Saúde Coletiva da UFTM, Uberaba, MG Brasil. ORCID: 0000-0002-0793-2594 E-mail: rosimarquerino@hotmail.com

6. Cientista Social. Mestre em Sociologia. Doutor e Pós Doutor em Ciências da Saúde. Professor Adjunto do Departamento de Saúde Coletiva da UFTM. ORCID: 0000-0002-7863-1211 E-mail: ailton.aragao@dms.uftm.edu.br 


\section{INTRODUCĈ̃O}

$\mathrm{O}$ trabalho é a expressão da condição humana, pois por meio dele, o homem, em relação social com outros homens, interfere na natureza e transforma, de modo dialético, a si e à natureza ${ }^{1}$. Na sociedade capitalista, o processo de trabalho gera não somente as condições de existência do trabalhador, mas também qualidade de vida e saúde ${ }^{2}$.

Neste contexto, um dos indicadores utilizados é a capacidade para o trabalho, que pode ser definida pela aptidão para executar funções, de acordo com as exigências laborais, estado de saúde, habilidades e condições físicas e mentais. É resultante da interação entre os recursos do indivíduo em relação a seu trabalho, e pode sofrer influência das condições em que o trabalho é exercido, da presença de morbidades e processos saúdedoença, e outros ${ }^{3-5}$.

A capacidade para o trabalhado associa-se, também, à qualidade de vida, entendida como a percepção do indivíduo de sua posição na vida, no contexto da cultura e sistema de valores nos quais ele vive, e em relação aos seus objetivos, expectativas, padrões e preocupações ${ }^{6}$.

Estudos têm revelado que quanto melhor a capacidade para o trabalho, maiores são os escores de qualidade de vida dos trabalhadores ${ }^{4,7-9}$. Um exemplo de instrumento validado e internacionalmente utilizado para avaliação da qualidade de vida é o Medical Outcomes Study 36 (SF-36), que foi traduzido e validado também para o português ${ }^{6}$.

A relação entre a capacidade para o trabalho e a qualidade de vida apresenta melhor associação com o domínio físico ${ }^{4}$. A capacidade de trabalho tem se mostrado fortemente associada à qualidade e segurança do ambiente de trabalho, gestão do tempo de lazer, níveis de absenteísmo por doença e custos financeiros para a empresa $8,10,11$.

A idade, sexo, renda e tempo de trabalho também predizem significativamente os níveis de qualidade de vida e capacidade para o trabalho ${ }^{10-13}$. Assim, considerando-se a amplitude e complexidade que envolve o constructo da qualidade de vida, destaca-se a necessidade de desenvolver programas especiais de promoção da saúde e prevenção de doenças, visando gerar melhorias na capacidade para o trabalho.

Neste contexto, esta pesquisa tem como objetivo avaliar a capacidade para o trabalho e qualidade de vida de trabalhadores atendidos pela rede pública municipal de saúde de Uberaba-MG, e identificar possíveis associações entre capacidade para o trabalho, qualidade de vida e características sociodemográficas, ocupacionais.

\section{MÉTODO}

Trata-se de um estudo do tipo observacional e transversal, que avaliou trabalhadores em acompanhamento na rede pública municipal de saúde da cidade de Uberaba-MG, no período de março a outubro de 2013.

Os critérios de inclusão utilizados foram: ser adulto (com idade entre 18 e 60 anos), de ambos os sexos, trabalhador, independente da situação atual no mercado de trabalho (ativo, afastado ou desempregado) e do tipo de contrato (formal ou informal), e estar em atendimento em uma das unidades de saúde municipais. Não foram incluídos os trabalhadores sem disponibilidade para participar do estudo, que apresentaram condições que impediam a compreensão ou fidedignidade das informações relatadas, ou que nunca desenvolveram atividades de trabalho.

Para estimar o tamanho da amostra considerou-se um nível de confiança de 95\%, erro máximo desejado de três pontos, e um desvio-padrão médio, estimado em um estudo piloto, igual a 15,5 pontos, totalizando uma amostra mínima de 103 indivíduos.

O sistema de saúde em Uberaba está dividido em três Distritos Sanitários e a atenção primária está organizada em 17 Unidades de Saúde de Família, 2 Unidades Básicas e 9 Unidades Matriciais de Saúde ${ }^{14}$. Os trabalhadores foram selecionados por conveniência em 9 unidades de saúde (Básicas e da Família) sorteadas aleatoriamente, respeitando o mínimo de três unidades por distrito sanitário e a avaliação dos 
participantes ocorreu no momento da sua consulta.

A caracterização sociodemográfica, econômica e ocupacional dos participantes ocorreu por meio da aplicação de questionário composto pelos itens sexo, idade, estado civil, escolaridade, renda, ocupação atual e tempo na ocupação, situação atual de trabalho (ativo, afastado ou desempregado) e, para os que estavam afastados, o tempo de afastamento.

Para avaliar a qualidade de vida empregou-se o instrumento validado SF-36 ${ }^{6}$. O SF-36 é um questionário composto por 36 perguntas que estão englobadas em oito domínios, a saber: capacidade funcional, aspectos físicos, dor, estado geral de saúde, vitalidade, aspectos sociais, aspecto emocional e saúde mental. Cada domínio possui um escore que varia de 0 (zero) a 100 (cem), sendo que quanto mais próximo do 0 , pior é a qualidade de vida e, quanto mais próximo de 100 , melhor é a qualidade de vida ${ }^{6}$.

Já a avaliação de capacidade para o trabalho foi realizada através do Índice de Capacidade para o Trabalho (ICT), que considera as demandas físicas e mentais, os recursos e a condição de saúde dos trabalhadores, segundo sua percepção. Os itens do ICT compõem sete dimensões: capacidade para o trabalho comparada com a melhor de toda vida, capacidade para o trabalho em relação a exigências físicas, número de doenças atuais diagnosticadas pelo médico, perda estimada para o trabalho por causa de doenças, faltas ao trabalho por doenças no último ano (12 meses), prognóstico próprio da capacidade para o trabalho daqui a 2 anos e recursos mentais ${ }^{3,12}$. Seu escore varia entre 7 e 49 pontos, sendo classificado em quatro níveis: capacidade para o trabalho baixa (7 a 27 pontos), capacidade moderada (28 a 36 pontos), capacidade boa (37 a 43 pontos) e capacidade ótima ( 44 a 49 pontos) $)^{3}$.
As análises estatísticas dos dados foram realizadas por meio do software estatístico Statistical Package for the Social Sciences (SPSS) para Windows, versão 20.0. Fizeram parte do banco de dados as variáveis sociodemográficas, econômicas e ocupacionais, devidamente codificadas, e as respostas dadas para cada item do ICT e da qualidade de vida. $\mathrm{Na}$ análise dos dados empregou-se, inicialmente, a estatística descritiva. Para associação das variáveis sociodemográficas, econômicas e ocupacionais e qualidade de vida com capacidade para o trabalho foram utilizados o teste Mann-Whitney $U$ e coeficiente de correlação de Spearman. Em todos os testes foi ponderado um nível de significância de $5 \%$. 0 estudo foi aprovado pelo Comitê de Ética em Pesquisa com Seres Humanos (parecer no 2394, de 23/02/2013).

\section{RESULTADOS}

Participaram deste estudo 111 trabalhadores, sendo a maioria do sexo feminino $(63,1 \%)$, idade média de 36,5 anos $(\mathrm{DP}=13,4)$ e renda mensal de $\mathrm{R} \$ 2.845,14$ ( $\mathrm{DP}=2.195,54)$, o que corresponde a 4,2 salários mínimos, como na Tabela 1.

A maior parte da amostra era composta de solteiros $(48,6 \%)$ e de participantes com ensino médio completo (33,3\%). 84,7\% dos participantes possuíam mais de 8 anos de estudo. Com relação à situação atual de trabalho, verificou-se que a maioria está ativa $(69,4 \%)$ e $30,6 \%$ encontram-se afastados. Entre os trabalhadores ativos, o tempo na ocupação variou entre um mês e 37 anos (média $=4,44$ anos e $\mathrm{DP}=6,4)$. Já no grupo de trabalhadores fora do mercado de trabalho, o tempo de afastamento foi em média 9,5 meses ( $D P=19,18$ meses), variando de um mês a oito anos, o que pode revelar uma incapacidade temporária ou até mesmo permanente para o trabalho (Tabela 1). 
Tabela 1. Características sociodemográficas e ocupacionais de trabalhadores atendidos pela rede pública de saúde. Uberaba-MG, 2013.

\begin{tabular}{|c|c|c|}
\hline Variáveis & Frequência absoluta (n) & Frequência relativa (\%) \\
\hline \multicolumn{3}{|l|}{ Sexo } \\
\hline Feminino & 70 & 63,1 \\
\hline Masculino & 41 & 36,9 \\
\hline \multicolumn{3}{|l|}{ Idade } \\
\hline Até 44 anos & 72 & 64,9 \\
\hline 45 anos ou mais & 39 & 35,1 \\
\hline \multicolumn{3}{|l|}{ Escolaridade } \\
\hline Ensino fundamental incompleto & 07 & 6,3 \\
\hline Ensino fundamental completo & 10 & 9,0 \\
\hline Ensino médio incompleto & 04 & 3,6 \\
\hline Ensino médio completo & 37 & 33,3 \\
\hline Ensino superior incompleto & 28 & 25,2 \\
\hline Ensino superior completo & 18 & 16,2 \\
\hline Pós-graduação & 07 & 6,3 \\
\hline \multicolumn{3}{|l|}{ Escolaridade agrupada } \\
\hline Até 8 anos de estudo & 17 & 15,3 \\
\hline Mais de 8 anos de estudo & 94 & 84,7 \\
\hline \multicolumn{3}{|l|}{ Estado civil } \\
\hline Solteiro & 54 & 48,6 \\
\hline Casado & 46 & 41,4 \\
\hline Separado/divorciado & 10 & 9,0 \\
\hline Viúvo & 01 & 0,9 \\
\hline \multicolumn{3}{|l|}{ Renda } \\
\hline Até 1 salário mínimo & 6 & 5,4 \\
\hline De 1 a 2 salários mínimos & 28 & 25,2 \\
\hline De 2 a 3 salários mínimos & 21 & 18,9 \\
\hline De 3 a 4 salários mínimos & 11 & 9,9 \\
\hline De 4 a 5 salários mínimos & 12 & 10,8 \\
\hline Acima de 5 salários mínimos & 33 & 29,7 \\
\hline \multicolumn{3}{|l|}{ Situação atual de trabalho } \\
\hline Ativo & 77 & 69,4 \\
\hline Afastado & 34 & 30,6 \\
\hline
\end{tabular}

Quanto à qualidade de vida, o domínio (média=61,6 e DP=42,0) apresentaram no qual os trabalhadores apresentaram melhores escores foi a capacidade funcional (média $=85,5 ; \quad \mathrm{DP}=17,1)$. Aspecto social (média $=76,0 ; \mathrm{DP}=27,1$ ), estado geral de saúde (média $=74,2 ; \mathrm{DP}=18,6$ ), dor (média $=71,0$; $\mathrm{DP}=24,4$ ) e saúde mental (média=70,2 e $\mathrm{DP}=15,4$ ) apresentaram valores moderados para qualidade de vida. Os domínios aspecto físico (média=69,1; DP=38,7), vitalidade (média=65,9; DP=17,2) e aspecto emocional valores que indicam pior qualidade de vida.

$\mathrm{Na}$ avaliação do ICT, os trabalhadores apresentaram em média o valor de 39,48 $(\mathrm{DP}=6,76)$. De forma categorizada, 10 trabalhadores $(8,1 \%)$ foram avaliados com baixa capacidade para o trabalho, $18(16,3 \%)$ com capacidade moderada, 58 (52,7\%) foram avaliados com boa capacidade, e 25 (22,7\%) atingiram escores que classificam a capacidade para o trabalho como ótima (Figura 1). 
Figura 1. Capacidade para o trabalho de trabalhadores atendidos pela rede municipal de saúde (n=111), Uberaba-MG, 2013.

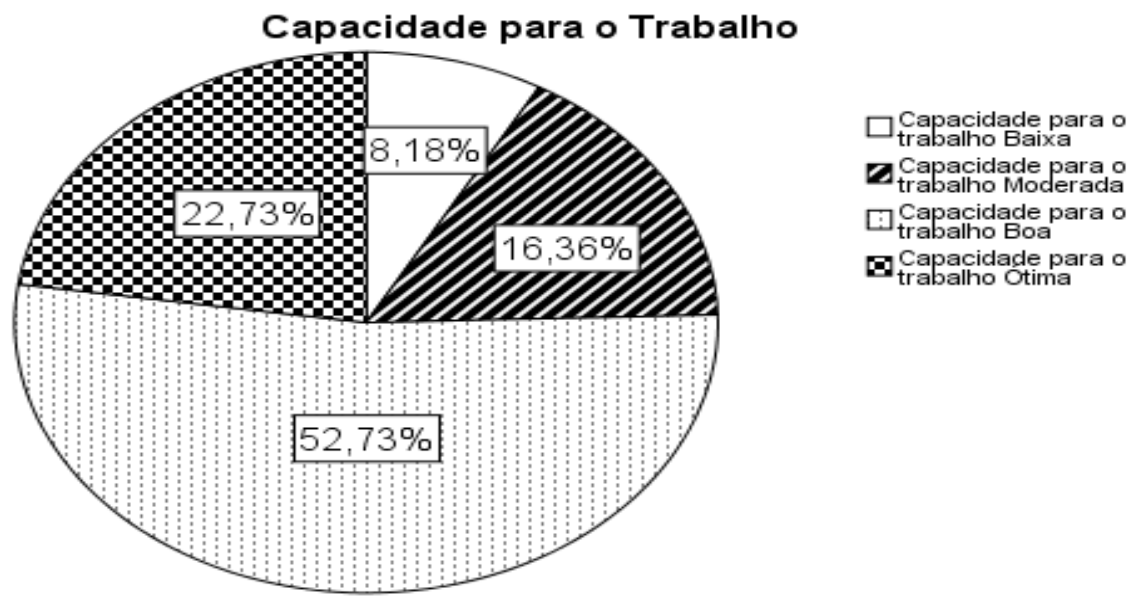

Os testes de associação entre as

e as variáveis gênero $(\mathrm{p}=0,209)$, renda variáveis sociodemográficas e ocupacionais indicaram relação significativa da capacidade para o trabalho com idade $(r=-0,286$; $(\mathrm{r}=0,182 ; \mathrm{p}=0,057)$, estado civil $(\mathrm{p}=0,844)$ e tempo no trabalho $(r=0,106 ; p=0,274)$. As $\mathrm{p}=0,002), \quad$ escolaridade $\quad(\mathrm{p}=0,000)$, tabelas 2 e 3 apresentam a descrição completa afastamento $(\mathrm{p}=000)$ e tempo de afastamento $(\mathrm{r}=-0,457 ; \mathrm{p}=0,000)$. Não houve diferença dos resultados da associação entre as variáveis sociodemográficas e ocupacionais com a capacidade para o trabalho. significativa entre capacidade para o trabalho

Tabela 2. Comparação entre capacidade para o trabalho e variáveis sociodemográficas e ocupacionais de trabalhadores atendidos pela rede municipal de saúde $(n=111)$, Uberaba-MG, 2013.

\begin{tabular}{llcc}
\hline \multicolumn{1}{c}{ Variáveis } & & \multicolumn{1}{c}{$\begin{array}{c}\text { Capacidade para o } \\
\text { trabalho (n=111) }\end{array}$} & Valor p \\
\cline { 2 - 3 } & & Média (DP) & \multirow{2}{*}{0,209} \\
\hline \multirow{2}{*}{ Sexo } & Feminino & $39,90(7,07)$ & \\
& Masculino & $38,73(6,20)$ & 0,844 \\
\hline \multirow{2}{*}{ Estado Civil } & Com companheiro (a) & $39,54(6,57)$ & 0,000 \\
\hline Escolaridade & Sem companheiro (a) & $39,43(6,95)$ & 0,000 \\
\hline Situação Atual em Relação & Ativo & $34,44(9,51)$ & $40,76(5,25)$ \\
ao Trabalho & Afastado & $41,45(4,34)$ & $35,07(8,90)$ \\
\hline
\end{tabular}

O ICT mostrou-se significativamente associado com todos os domínios da qualidade de vida: capacidade funcional $(\mathrm{p}=0,000 ; \mathrm{r}=0,461)$, limitação por aspectos físicos ( $\mathrm{p}=0,000 ; \mathrm{r}=0,587)$, dor $(\mathrm{p}<0,01$; $r=0,47)$, estado geral de saúde $(p<0,01$; $\mathrm{r}=0,49)$, vitalidade $(\mathrm{p}<0,01 ; \mathrm{r}=0,25)$, aspectos sociais $(p<0,01 ; r=0,61)$ e saúde mental $(\mathrm{p}<0,05, \mathrm{r}=0,24)$. Estes resultados indicaram que quanto maior a qualidade de vida nestes domínios, melhor era a capacidade para o trabalho dos indivíduos avaliados (Tabela 3). 
Tabela 3. Correlação entre capacidade para o trabalho e variáveis sociodemográficas, ocupacionais e de qualidade de vida de trabalhadores atendidos pela rede municipal de saúde (n=111), Uberaba-MG, 2013.

\begin{tabular}{llcc}
\hline \multirow{2}{*}{ Variáveis } & & \multicolumn{2}{c}{ Capacidade para o Trabalho } \\
\cline { 3 - 4 } Sócio Demográficas & Idade & $\mathbf{r}$ & Valor p \\
\cline { 2 - 3 } Ocupacionais & Renda & $-0,286$ & 0,002 \\
& & 0,182 & 0,057 \\
Qualidade de Vida & & \\
& Tempo no Trabalho & 0,106 & 0,274 \\
& Tempo de Afastamento & $-0,457$ & 0,000 \\
& Capacidade Funcional & & \\
& Limitação por Aspectos Físicos & 0,461 & 0,000 \\
& Dor & 0,587 & 0,000 \\
& Estado Geral de Saúde & 0,528 & 0,000 \\
& Vitalidade & 0,469 & 0,000 \\
& Aspectos Sociais & 0,338 & 0,000 \\
& Aspectos Emocionais & 0,498 & 0,000 \\
& Saúde Mental & 0,462 & 0,000 \\
& & 0,373 & 0,000 \\
\hline
\end{tabular}

\section{DISCUSSÃo}

Os resultados encontrados apontam para uma associação entre capacidade para o trabalho e uma melhor qualidade de vida dos trabalhadores atendidos na atenção primária. Entre os participantes, observou-se a predominância de mulheres, o que pode estar associado ao padrão de procura e de uso dos serviços de saúde entre a população brasileira. A frequência de busca por serviços nas unidades básicas saúde é predominante entre a população feminina e atribuída a fatores como reprodutividade, idade, pior percepção de saúde, bem como maior incidência de doenças crônicas não-fatais ${ }^{15}$.

Em relação a renda e anos de estudo, a população de estudo é favoravelmente diferenciada em relação à população geral de trabalhadores, com maior faixa de rendimentos e nível de escolaridade ${ }^{16}$. No município de Uberaba/MG, a maioria dos estabelecimentos de saúde que oferece atenção primária pertence ao serviço público. Assim, o acesso a determinados serviços de saúde por meio das unidades básicas são as opções de atendimento ofertadas para todas as faixas de renda da população ${ }^{17}$.

Destaca-se que, entre os participantes, havia um grande número de trabalhadores afastados de suas atividades laborais por um período de tempo relativamente longo. Os trabalhadores afastados tendem a pior qualidade de vida e pior percepção de saúde quando comparados aos trabalhadores ativos no mercado de trabalho ${ }^{18}$, reforçando a importância de ações e políticas para inserção de indivíduos adultos em atividades laborais. Neste sentido, os serviços públicos de saúde devem se mobilizar para proporcionar meios de assistência e de tratamento para que os trabalhadores afastados possam retornar ao trabalho.

Para a promoção e a manutenção da qualidade de vida dos indivíduos torna-se fundamental a adoção de medidas preventivas e efetivas de acesso a empregos e intervenções que minimizem a ocorrência de afastamento do trabalho ${ }^{18}$. Entretanto, o Sistema Único de Saúde ainda dispõe de poucos serviços de reabilitação profissional e as políticas públicas voltadas à saúde do trabalhador ainda precisam ser fortalecidas para garantir o atendimento integral em saúde ${ }^{19}$.

Quando se analisa os resultados especificamente relacionados à qualidade de vida da população, observam-se também médias mais baixas, principalmente nos domínios relacionados aos aspectos físico, vitalidade e aspectos emocionais, em 
comparação com a pontuação da população de trabalhadores de outros estudos brasileiros ${ }^{20,21}$. Estes resultados não são esperados para uma população de usuários da atenção básica. 0 que pode indicar a necessidade de se avaliar com mais detalhamento a saúde e os fatores associados à qualidade de vida entre os trabalhadores de Uberaba.

Quanto à capacidade para o trabalho, verificou-se que apenas $75 \%$ dos trabalhadores foram classificados como tendo boa e excelente capacidade para o trabalho, com uma média de 38,5 para o ICT. Estes resultados são piores do que os apresentados por trabalhadores de setores da indústria ${ }^{4}$, da saúde $^{10,22}$, do vestuário ${ }^{23}$ e da educação ${ }^{24}$.Em sentido amplo a capacidade para o trabalho é entendida como todas as capacidades necessárias à execução de um determinado tipo de trabalho e, em um sentido restrito, expressa aptidão para o trabalho. Na prática representam combinações entre recursos humanos em relação às demandas físicas, mentais e sociais do trabalho, cultura organizacional e ambiente de trabalho ${ }^{11}$.

Levando em consideração estes resultados descritivos da capacidade para o trabalho, é importante o levantamento do perfil ocupacional da população atendida pelas unidades de saúde analisadas. A capacidade para o trabalho ruim ou em declínio está associada ao desenvolvimento de condições de saúde relacionadas a desordens psicossomáticas ${ }^{24}$, transtornos mentais $^{25-27}$ e disfunções físicas ${ }^{28,29}$.

Assim, o levantamento do perfil ocupacional da população adstrita pode auxiliar no diagnóstico e tratamento das doenças relacionadas ao trabalho e no estabelecimento da relação entre declínio da CT com o adoecimento do trabalhador. Em acréscimo, este tipo de ação desenvolvida pelas equipes de saúde da atenção básica pode culminar em parcerias intersetoriais com os Centros de Referência em Saúde do Trabalhador (CEREST) e Instituto Nacional do Seguro Social (INSS), orientando e encaminhando trabalhadores para o provimento dos benefícios previdenciários correspondentes.
Houve associação da capacidade para o trabalho com idade, escolaridade e tempo de afastamento do trabalho, corroborando outros estudos realizados no Brasil11,30,31. A idade dos participantes variou pouco, com mais de $70 \%$ da amostra composta por trabalhadores com até 44 anos e confirmando o declínio da capacidade para o trabalho a partir dos 45 anos já indicado na literatura11,30,31. Pesquisas indicam associação positiva entre escolaridade e capacidade para o trabalho ${ }^{32,33}$, confirmando os resultados apresentados que mostram pior capacidade para o trabalho em profissionais com baixa escolaridade (menos de oito anos de estudo). Em geral, maior escolaridade está relacionada a probabilidade de inserção em postos de trabalho mais qualificados e menos agressivos à saúde ${ }^{33}$.

$$
\text { A dimensão emocional foi }
$$
diagnosticada como o pior domínio da qualidade de vida. As consequências adversas relacionadas à saúde, condições precárias de trabalho ou desemprego podem acarretar a desestruturação de laços sociais e afetivos, a restrição de direitos, a insegurança socioeconômica, a redução da autoestima, o sentimento de solidão e fracasso, o desenvolvimento de distúrbios mentais, bem como o aumento do consumo ou dependência de drogas ${ }^{34}$.

De modo geral, os estudos sobre saúde mental no trabalho, bem como os dados epidemiológicos e previdenciários, têm mostrado crescimento do número de transtornos mentais e do comportamento relacionados ao trabalho e à crescente diminuição da qualidade de vida emocional dos trabalhalhadores ${ }^{2}$. A relação entre essas variáveis também foi investigada em outro estudo $^{35}$ que obteve resultados semelhantes, mostrando que quanto maiores são os níveis percebidos de qualidade de vida, menor é o estresse percebido e melhor é a capacidade para o trabalho.

Aspectos emocionais, sociais e de saúde mental foram componentes da qualidade de vida pior avaliados em trabalhadores afastados do trabalho do que em trabalhadores ativos no emprego em outro estudo realizado com usuários da atenção 
básica ${ }^{18}$. 0 trabalho constituiu-se para o homem como um meio rico de produção da vida de cada um, criando sentidos existenciais ou contribuindo na estruturação da personalidade e da identidade ${ }^{34}$.

Neste sentido, o desempregado, aquele que vivencia a falta de renda, a exclusão do mundo do trabalho e que vive à margem da sociedade, deve ser visto de forma atenta pelos profissionais da área da saúde buscando intervir nas reações simbólicas e materiais causadas pelo afastamento do trabalho, contribuindo desse modo para prevenir e amenizar agravos à saúde física e mental advindos dessa situação ${ }^{34}$.

O domínio da qualidade de vida relacionado com a capacidade funcional também apresentou correlação significativa com a capacidade para o trabalho. Esta correlação da capacidade para o trabalho com a capacidade funcional aponta que, além da saúde, há outro determinante importante para capacidade para o trabalho. A capacidade funcional é considerada a habilidade do indivíduo em realizar atividades do seu cotidiano; garantindo sua autonomia.

Um estudo realizado com esta mesma população confirma a correlação entre capacidade para o trabalho e capacidade funcional, apontando que trabalhadores com melhor funcionalidade apresentam melhor capacidade para o trabalho ${ }^{36}$. Tal associação também foi observada em outro estudo que indica a relação entre a menor percepção de capacidade para o trabalho e menor percepção de capacidade funcional ${ }^{20}$. Por seu turno, um estudo 37 mostrou que a capacidade funcional e a capacidade para o trabalho em pessoas com dor musculoesquelética crônica, têm forte correlação, de modo que a capacidade funcional é muito importante na prevenção de incapacidades para o trabalho.

$\mathrm{Na}$ capacidade para o trabalho e nos domínios da qualidade de vida, o aspecto físico mostrou correlação significativa de magnitude forte. De forma geral, o declínio da saúde pode restringir a capacidade física e funcional e, consequentemente, afetar a capacidade de trabalho. Esse resultado ratifica os achados de outros estudos em que as características do trabalho e suas demandas físicas impactaram de forma negativa a capacidade do trabalhador responder a estas exigências físicas $7,23,30,38$. Assim, funções com alta exigência física têm sido associadas a pior capacidade para o trabalho $22,29,37$ e reforçam a necessidade do desenvolvimento de ações preventivas direcionadas aos aspectos ligados ao domínio físico.

O domínio dor também apresentou impacto significativo sobre a capacidade para o trabalho. Esse resultado é consonante com outra pesquisa que mostra que a dor crônica muscular reduziu a capacidade para o trabalho de uma amostra de trabalhadoras de três cidades metropolitanas da Suécia ${ }^{39}$.

Outro estudo ${ }^{30}$ acompanhou a capacidade para o trabalho de funcionários de uma indústria e encontrou correlação negativa e moderada de dor musculoesquelética com a capacidade de trabalho, indicando uma influência da presença e da intensidade da dor na determinação da atual CT. Em contrapartida, intervenções, baseadas em treinamentos de força muscular contribuem para a redução da dor e o número de licenças médicas ${ }^{39}$, o que também corrobora a importância de ações preventivas voltadas para a capacidade funcional dos trabalhadores.

Nesse cenário, os programas de intervenção voltados à melhora na capacidade para o trabalho e qualidade de vida são essenciais para a manutenção da produtividade das organizações de trabalho, para a diminuição dos índices de afastamentos no trabalho e prevenção de doenças $9,13,23$. Os resultados apontam para uma relação entre capacidade para o trabalho e qualidade de vida e indicam ser fundamental o desenvolvimento de atividades que promovam a saúde e qualidade de vida dos trabalhadores.

Boas condições de trabalho aliadas ao acesso à educação pelo trabalhador, ainda na adolescência, devem ser implementadas para eliminar os fatores que impactarão negativamente sobre a capacidade para o trabalho ${ }^{23}$. Estas iniciativas incluem, por exemplo, aspectos ergonômicos, de modo a prevenir morbidades, associados, ainda, a 
melhores condições estruturais e ambientais desses mesmos ambientes de trabalho.

Além disso, é necessário que o trabalhador tenha acesso à formação profissional oferecida pelo setor público, a partir do financiamento de cursos técnicos, ações educativas de iniciação ao trabalho e de especialização, por meio do Ministério da Educação, Escolas Técnicas Estaduais e Municipais, Escolas Públicas, Universidades, Ministério do Trabalho e outras ${ }^{19,40}$.

\section{CONCLUSÃO}

Os resultados obtidos indicam que a capacidade para o trabalho está significativamente correlacionada com os domínios da qualidade de vida. 0 estudo demonstrou uma correlação significativa entre gênero, escolaridade e tempo de afastamento com a capacidade para o trabalho. Este resultado corrobora o arcabouço teórico acerca da capacidade para o trabalho, incorporando em sua análise uma perspectiva multifatorial e multidimensional.

Este estudo apresenta algumas características que podem ser limitantes, como o delineamento transversal, apresentando resultados que indicam associação entre qualidade de vida $\mathrm{e}$ capacidade para o trabalho. No entanto, não se pode estabelecer uma relação causal entre as variáveis investigadas. Estudos de acompanhamento longitudinal da capacidade para o trabalho devem ser delineados juntamente com a avaliação de fatores associados. A caracterização da amostra mostrou escolaridade e renda acima da média da população brasileira atendida por serviços públicos de saúde. Assim, generalizações para outros estudos e populações devem ser feitas com prudência.

Apesar destas limitações, este estudo explora a relação entre capacidade para o trabalho e qualidade de vida na população geral adulta atendida por um serviço de atenção básica. Estas informações podem subsidiar ações de vigilância epidemiológica, e em saúde do trabalhador voltada para intervenções no nível primário de cuidado em saúde. Seus resultados podem subsidiar a atuação de equipes multiprofissionais na implementação de estratégias de promoção da saúde e da qualidade de vida, tanto individual quanto coletivamente.

\section{REFERÊNCIAS}

1. Bendassolli PF, Gondim SMG. Significados, sentidos e função psicológica do trabalho: Discutindo essa tríade conceitual e seus desafios metodológicos: explorando conceitos, variáveis e estudos empíricos brasileiros. AvPsicol Latinoam. 2014; 32(1):131-47.

2. Souza KR, Rodrigues AMS, Fernandez VS, Bonfatti RJ. A categoria saúde na perspectiva da saúde do trabalhador: ensaio sobre interações, resistências e práxis. Saúde Debate. 2017; 41(esp2):254-63.

3. Tuomi K, Ilmarinen J, Jahkola A, Katajarinne L, Tulkki A. Índice de capacidade para o trabalho. São Carlos: EduFSCar; 2005.

4. Costa CSN, Freitas EG, Mendonça LCS, Alem MER, Coury HJCG. Capacidade para o trabalho e qualidade de vida de trabalhadores industriais. Ciênc Saúde Colet. 2012; 17(6):1635-42.

5. Valinote HC, Pacheco LF, Viana FP, Formiga CKMR. Análise da qualidade de vida, capacidade para o trabalho e nível de estresse em trabalhadores da construção civil. Rev Bras Ciênc Ambient. 2014; (32):115-26.

6. Ciconelli RM, Ferraz MB, Santos W, Meinão I, Quaresma MR. Tradução para a língua portuguesa e validação do questionário genérico de avaliação de qualidade de vida SF-36 (Brasil SF-36). Rev Bras Reumatol. 1999;39(3):143-50.

7. Tavakoli-Fard N, Mortazavi SA, Kuhpayehzadeh J, Nojomi M. Quality of life, work ability and other important indicators of women's occupational health. Int J Occup Med Environ Health. 2016;29(1):77-84.

8. Biallas B, Froböse I, Zöller M, Wilke C. Analysis of workplace health promotion and its effect on work ability and health-related quality of life in a medium-sized business. Gesundheitswesen. 2015;77(5):357-61.

9. Mcdonald M, Dibonaventura MD, Ullman S. Musculoskeletal pain in the workforce: the effects of back, arthritis, and fibromyalgia pain on quality of life and work productivity. J Occup Environ Med. 2011; 53(7):765-70.

10. Martinez MC, Latorre MRDO, Fischer FM.Testando o modelo da casa da capacidade para o trabalho entre profissionais do setor hospitalar. Rev Bras Epidemiol. 2016;19(2): 403-18.

11. Sampaio RF, AugustoVG. Envelhecimento e trabalho: um desafio para a agenda da 
reabilitação. Rev Bras Fisioter. 2012;16(2):94101.

12. Berg TIJ, Elders LAM, Zwart BCH, Burdorf A. The effects of work-related and individual factors on the work ability index: a systematic review. Occup Environ Med. 2009; 66(4):211-20.

13. Rothman MG, Ortendahl M, Rosenblad A, Johansson A. Improved quality of life, working ability, and patient satisfaction after a pretreatment multimodal assessment method in patients with mixed chronic muscular pain: a randomized-controlled study. Clin J Pain. 2013; 29(3):195-204.

14. Prefeitura Municipal de Uberaba. Plano Municipal de Saúde 2014-2017. Secretaria Municipal de Saúde - Assessoria de Planejamento em Saúde, 2014. 157p. [citado em 10 jun 2016]. Disponível

em:http://www.uberaba.mg.gov.br/portal/acerv o/saude/arquivos/plano_municipal_saude.pdf

15. Stopa SR, Malta DC, Monteiro CN, Szwarcwald CL, Goldbaum M, Galvão Cesar CL. Acesso e uso de serviços de saúde pela população brasileira, Pesquisa Nacional de Saúde 2013.Rev Saúde Pública. 2017;51(Supl1):1s-11s.

16. Instituto Brasileiro de Geografia e Estatística. Pesquisa mensal de emprego: rendimento real habitual do trabalho principal [Internet]. Rio de Janeiro: IBGE; 2013 [citado em 10 jun 2015]. Disponível em: http://www.ibge.gov.br/home/estatistica/indica dores/trabalhoerendimento/pme_nova/defaultta b_hist.shtm

17. Instituto Brasileiro de Geografia e Estatística. Censo 2010 [Internet]. Rio de Janeiro: IBGE; [2011-2015] [citado em 10 jun 2015]. Disponível em: http://censo2010.ibge.gov.br/.

18. Dutra FCMS, Costa LC, Sampaio RF. A influência do afastamento do trabalho na percepção de saúde e qualidade de vida de indivíduos adultos. Fisioter Pesqui. 2016;23(1):98-104.

19. Poersch AL, Merlo ARC. Reabilitação profissional e retorno ao trabalho: uma aposta de intervenção. Psicol Soc. 2017;29(149496):1-10.

20. Queiroz DL, Souza JC. Qualidade de vida e capacidade para o trabalho de profissionais de enfermagem. Psicol inF. 2012; 16(16):103-26.

21. Milosevic M, Golubic R, Knezevic B, Golubic K, Bubas M, Mustajbegovic J. Work ability a major determinant of clinical nurses' quality of life. J Clin Nurs. 2011; 20(19):2931-8.

22. Rostamabadi A, Zamanian Z, SedaghatZ. Factors associated with work ability index (WAI) among intensive care units' (ICUs') nurses. J Occup Health. 2017; 59:147-55.
23. Augusto VG, Sampaio RF, Ferreira FR, Kirkwood RN, Cesar CC. Factors associated with inadequate work ability among women in the clothing industry. Work. 2015; 50(2):275-83.

24. Santino TM, Tomaz AF, Lucena NMG. Influência da fadiga ocupacional na capacidade para o trabalho de professores universitários. Ciênc Trab. 2017;19(59):86-90.

25. Gould R, Ilmarinen J, Järvisalo J, Koskinen S, editors. Dimensions of work ability: results of the Health 2000 Survey. Finland: FIOH; 2008.

26. Magnago TSBS, Prochnow A, Urbanetto JS, Greco PBT, Beltrame M, Luz EMF. Relação entre capacidade par ao trabalho na enfermagem e distúrbios psíquicos menores. Texto\& Contexto Enferm. 2015; 24(2),262-70.

27. Carvalho DB, Araújo TM, Bernardes KO. Transtornos mentais comuns em trabalhadores da Atenção Básica à Saúde. Rev Bras Saúde Ocup. 2016; 41(17):1-13

28. Magnago TSBS, Lima ACS, Prochnow A. Intensidade da dor musculoesquelética e a (in)capacidade para o trabalho na enfermagem. Rev Latinoam Enferm. 2012; 20(6):3-9.

29. Boschman JS, Noor A, Lundstrom R, Nilsson T, Sluiter JK, Hagberg M. Relationships between work-related factors and musculoskeletal health with current and future work ability among male workers. Int Arch Occup Environ Health. 2017; 90(6):517-26.

30. Alcântara MA, Sampaio RF, Assunção AA, Silva FCM. Work Ability: using structural equation modeling to assess the effects of aging, health and work on the population of Brazilian municipal employees. Work 2014; 49(3):465-72.

30. Prochnow A, Magnago TSBS, Urbanetto JS, Beck CLC, Lima SBS, Greco PBT. Capacidade para o trabalho na enfermagem: relação com demandas psicológicas e controle sobre o trabalho. Rev Latinoam Enferm. 2013; 21(6):1298-305.

31. Martinez MC, Latorre MRDO, Fischer FM. Testando o modelo da casa da capacidade para o trabalho entre profissionais do setor hospitalar. Rev Bras Epidemiol. 2016; 19(2):403-18.

32. Cordeiro TMSC, Araújo TM. Capacidade para o trabalho entre trabalhadores do Brasil. Rev Bras Med Trab. 2016; 14(3):262-274.

33. Pinheiro LRS, Monteiro JK. Refletindo sobre desemprego e agravos à saúde mental. Cad Psicol Soc Trab. 2007;10(2):35-45.

34. Petroski EC. Qualidade de vida no trabalho e suas relações com estresse, nível de atividade física e risco coronariano de professores universitários. [Tese]. Florianópolis: Universidade Federal de Santa Catarina; 2013. 
35. Valério RBC, Dutra FCMS. Envejecimiento funcional y capacidad de trabajo entre los trabajadores atendidos en la atención primaria. Ciênc Trab. 2016;18(57):190-5.

36. Lillefjell M. Function and work ability following multidisciplinary habilitation for individuals with chronic musculoskeletal pain. [Thesis]. Norway: Faculty of Social Sciences and Technology Management; 2007.

37. Alcantara MA, Assuncão AA. Influência da organização do trabalho sobre a prevalência de transtornos mentais comuns dos agentes comunitários de saúde de Belo Horizonte. Rev BrasSaúde Ocup. 2016;41(e2).

38. Dellve L, Ahlstrom L, Jonsson A, Sandsjö L, Forsman M, Lindegård $A$, et al. Myofeedback training and intensive muscular strength training to decrease pain and improve work ability among female workers on long-term sick leave with neck pain: a randomized controlled trial. Int Arch Occup Environ Health. 2011; 84(3):335-46.

39. Schwartzman S, Castro C. Ensino, formação profissional e a questão da mão de obra. Ensaio: Aval Polít Públicas Educ. 2013; 21(80):563-624.

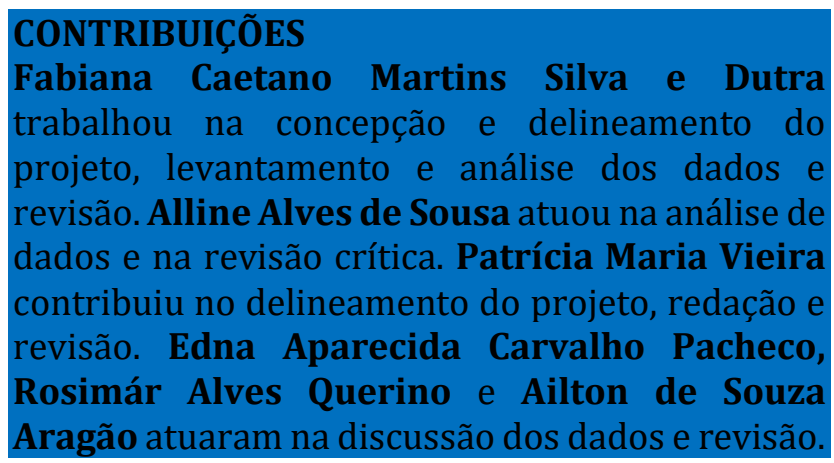

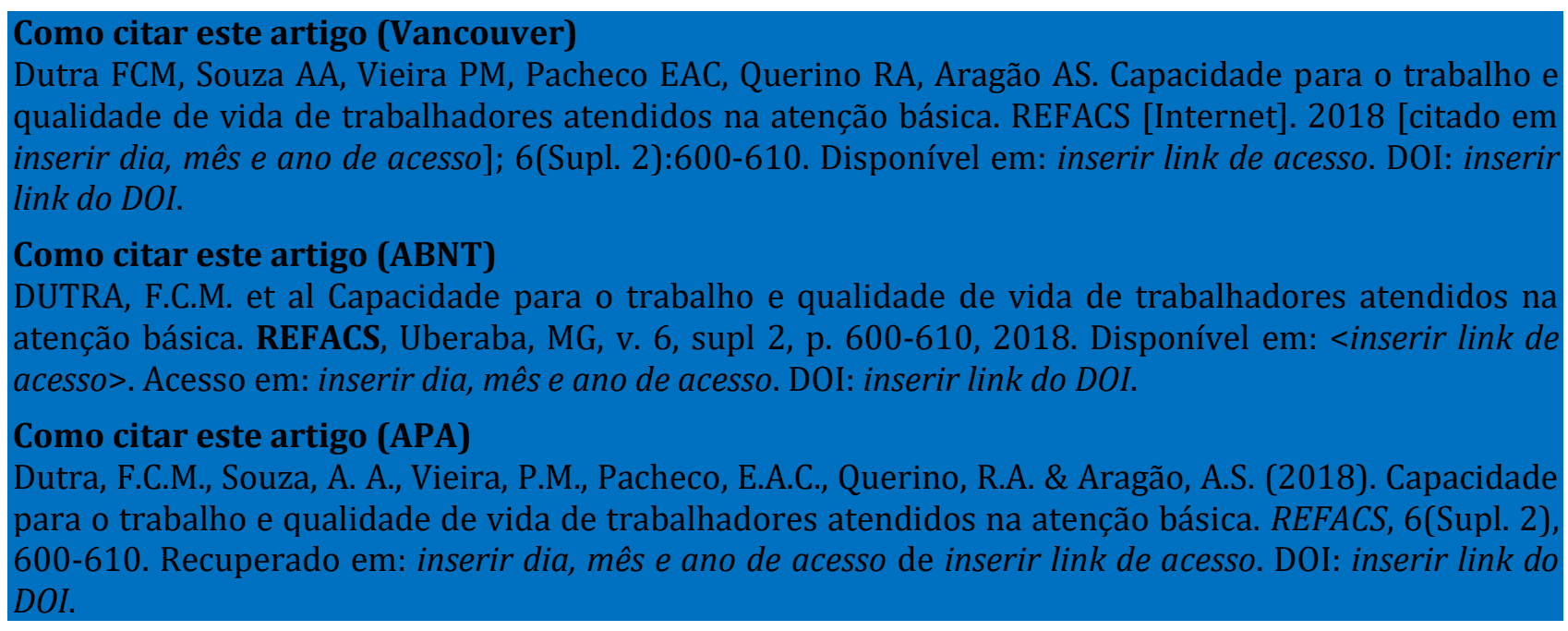

